\title{
Periarticular histiocytic sarcoma of a thoracic limb in a Rottweiler
}

\author{
Hyeok-Soo Shin ${ }^{1}$, Ye-In $\mathrm{Oh}^{2}$, Byung-Jae Kang ${ }^{1, *}$ \\ ${ }^{1}$ Department of Veterinary Surgery, College of Veterinary Medicine and Institute of Veterinary Science, \\ Kangwon National University, Chuncheon 24341, Korea \\ ${ }^{2}$ Department of Veterinary Internal Medicine, College of Veterinary Medicine, Seoul National University, Seoul 08826, Korea
}

(Received: January 2, 2018; Revised: February 21, 2018; Accepted: February 26, 2018)

\begin{abstract}
An 8-year-old, castrated, male Rottweiler was referred for evaluation of chronic right thoracic limb lameness and a progressively growing mass surrounding the right elbow joint. On admission, the dog's general health was good, without abnormalities detected on physical examination. The dog was diagnosed with periarticular histiocytic sarcoma. Although draining lymph nodes and lung metastases were suspected, palliative amputation was performed. Localized histiocytic sarcomas, with destructive lesions involving multiple bones of a joint and periarticular soft-tissue masses, are uncommon in dogs. This case report presents clinical findings, imaging characteristics, and histopathologic and immunohistochemical features of a periarticular joint histiocytic sarcoma.
\end{abstract}

Keywords: bone, dogs, immunohistochemistry, periarticular histiocytic sarcoma

Histiocytes are derived from bone marrow stem cells and can be either macrophages or dendritic cells. Dendritic cells can be further subdivided into Langerhans cells found in the skin, interstitial dendritic cells found in many organ systems, and interdigitating dendritic cells found in the $\mathrm{T}$ cell zone. Because of these various differentiation potentials of histiocytes, canine histiocytic proliferative disorders represent a diagnostic and therapeutic challenge [11, 12, 14].

Various canine histiocytic proliferative diseases have been reported in dogs, including reactive diseases such as cutaneous and systemic histiocytosis. Neoplastic histiocytic diseases are classified into histiocytomas (benign forms) or histiocytic sarcomas (HSs; malignant forms). HSs are further classified into the disseminated or localized form. Localized HS involves a single primary lesion, whereas disseminated HS involves multiple organ systems [2] and presents multiple tumor masses in several organ systems. Primary lesions are usually seen in the bone marrow, spleen, and lung, whereas secondary lesions are observed in the liver, lymph nodes, and sometimes other organs. Dogs with disseminated HS often show anorexia, weight loss, and lethargy [2, 15]. Localized HSs arise from a single organ. They are locally invasive and can metastasize to regional lymph nodes. The subcutis is a major invasion site, but other primary sites have been observed in the stomach, lungs, liver, spleen, pancreas, and central nervous system. A more favorable prognosis can be expected for dogs with localized HS than with dissemi- nated HS, which is an invasive multiorgan disorder with a grave prognosis [2]. The etiology of HS is unknown, but a clear genetic link has been observed. Rottweiler, Golden Retriever, Labrador Retriever, Doberman Pincher, and Bernese Mountain Dog are predisposed breeds, and middle- to oldaged dogs are often affected [1, 5, 13].

Dogs with localized HS, which occurs at a focal limb, often present with lameness associated with one joint because the tumor can arise from the dendritic cells of the synovial lining. Moreover, the tumor can affect the surrounding bone and soft tissue [4]. The Rottweiler, described herein, developed chronic lameness and pain due to periarticular histiocytic sarcoma (PAHS).

An 8-year-old, castrated, male Rottweiler, weighing $38 \mathrm{~kg}$, was referred for the evaluation of chronic right thoracic limb lameness and pain. Three months previously, mild soft-tissue swelling and pain in the region of the elbow joint was detected by the referring veterinarian and treated with nonsteroidal anti-inflammatory drugs, antibiotics, and restricted physical activity. However, the dog showed only temporary improvement. Over the previous month, the mass rapidly increased in size and the dog showed intermittent lameness in the right thoracic limb.

On admission, the dog showed good general health but had been showing mild anorexia for 1 week. Right axillary lymph node enlargement was identified on a physical examination. An orthopedic examination revealed intermittent lameness in

*Corresponding author

Tel: +82-33-250-8207, Fax: +82-33-259-5625

E-mail: bjkang@kangwon.ac.kr 

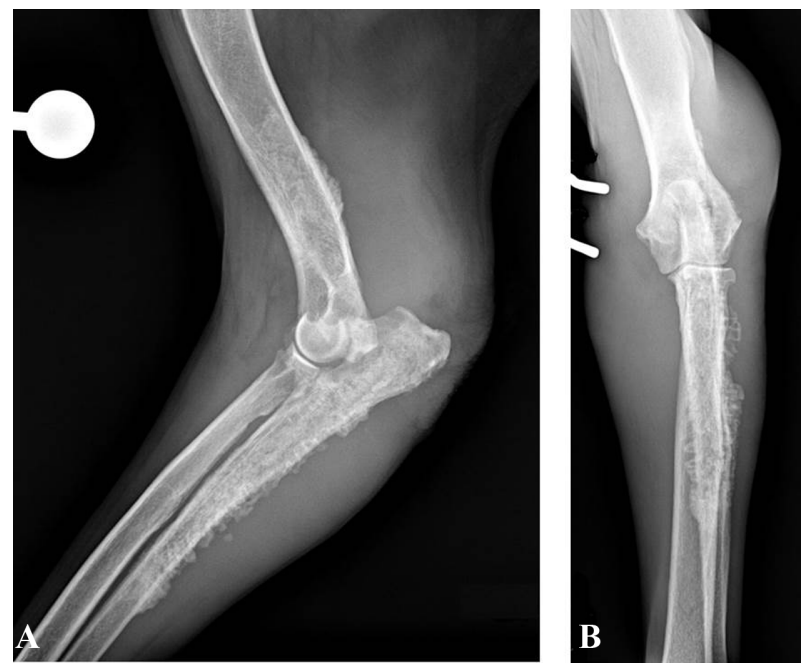

Fig. 1. Lateral (A) and dorsoventral (B) radiographs demonstrating a large soft-tissue mass surrounding the right elbow joint and irregular bone proliferation at the distal humerus, proximal radius, and ulna.

the right forelimb. The range of motion of the elbow joint was restricted to about $60 \%$ of that of the normal leg, and the dog showed pain when the affected elbow joint was manipulated. Radiography revealed a large soft-tissue mass around the elbow joint. Moreover, new bone production, cortical destruction, and perceptive lysis were present within the distal humerus and proximal ulna (Fig. 1). Neoplastic and infectious diseases were listed as the main causes. Fine-needle aspiration of the mass was performed under ultrasound guidance. Microscopic examination revealed marked cellular and nuclear pleomorphism and giant multinucleated cells, characteristic of a malignant tumor. In addition, the microscopic examination revealed the dominance of spindle cells, which are features of mesenchymal cell origin tumors. Microorganisms were not observed on synovial fluid examination, and a synovial culture showed no bacterial growth. A tentative diagnosis of a neoplastic disorder was made, and osteosarcoma, fibrosarcoma, chondrosarcoma, osteochondrosarcoma, synovial cell sarcoma, and HS were included in the differential diagnosis list. Computed tomography (CT) was performed to evaluate the metastasis and neoplastic change in the right periarticular region. A complete blood count, serum biochemical analysis, and thoracic radiography were performed prior to anesthesia, and these revealed only mild anemia. The iso- to hypo-attenuating mass was found near the right elbow joint on pre-contrast CT. In addition to the surrounding bone structure, the synovial membrane showed neoplastic changes (Fig. 2). The right axillary lymph node was enlarged $\left(6 \times 3 \mathrm{~cm}^{2}\right)$. Chest CT revealed multiple small metastatic pulmonary nodules. The prognosis was expected to be negative because of the suspicion of metastasis, the degree of pain worsened, and the size of the tumor increased; therefore, palliative amputation was considered. The right
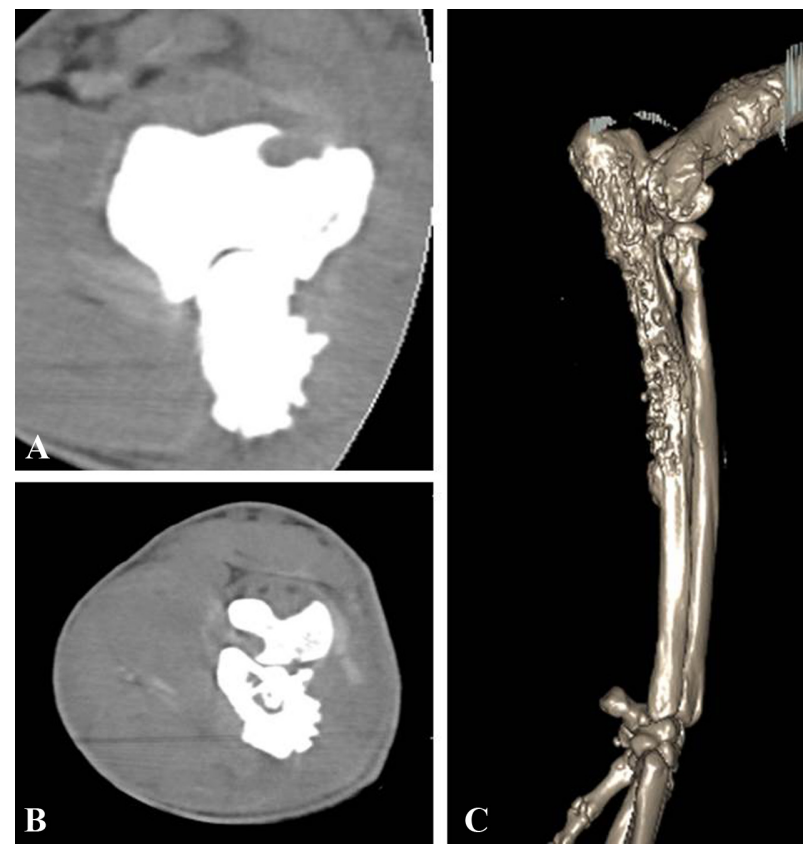

Fig. 2. Computed tomography images of the humeral condyle (A) and proximal radius and ulna (B) demonstrate the combination of irregular bone lysis and periosteal reaction. (C) Threedimensionally reconstructed image of the distal humerus, radius, and ulna in the lateral aspect.

thoracic limb was amputated through the removal of the scapula. Axillary lymph nodes and other regional lymph nodes were also removed during the surgery. The dog recovered without any complication and showed good general health. However, the owner refused the administration of adjuvant chemotherapy. The limb and removed lymph nodes were submitted for histopathologic evaluation. On gross examination, the bone marrow and bony structure of the proximal ulna and distal humerus were effaced by an unencapsulated, poorly circumscribed, infiltrative neoplasm. The large mass surrounding the elbow joint was due to the neoplastic proliferation of neighboring muscles and joint capsule. Furthermore, proliferated soft tissues were filled with a hemopurulent discharge. The bone neoplasm was composed of spindle cells and occasional round cells, sometimes arranged in pleomorphic sheets supported by existing stroma. Bone and axillary lymph node had the same features on microscopic observation. Neoplastic cells had distinct cell borders and moderate amount of amphophilic cytoplasm. Nuclei were round to oval and had finely to coarsely stippled chromatin and 1-3 indistinct nucleoli. Marked anisocytosis and anisokaryosis were seen. Marked osteolysis and mesenchymal proliferation were also seen (Fig. 3A). More than $90 \%$ of the local lymph nodes showed necrotic changes, and multiple aggregates of lymphocytes, plasma cells, and macrophages with fibrous tissue were seen at the periphery. To obtain a definitive diagnosis, immunohistochemical evaluation was performed, which revealed a large number of 

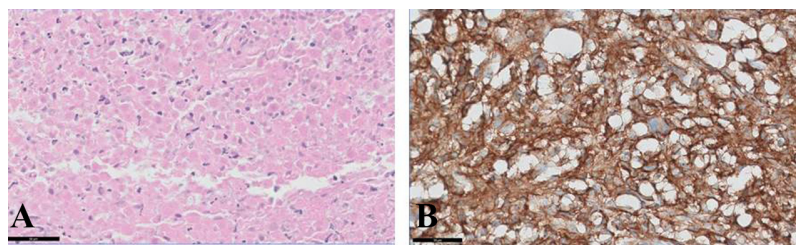

Fig. 3. (A) Histological appearance of the histiocytic sarcoma characterized by marked anisocytosis and anisokaryosis. H\&E stain. (B) Positive immunohistochemical staining of neoplastic cells for CD18. Scale bars $=50 \mu \mathrm{m}$.

CD18-positive cells and low to moderate number of vimentin-positive cells (Fig. 3B). On the basis of the histological criteria and immunohistochemical features, a final diagnosis of PAHS was made. The dog showed good general health during 4 months after the surgery, but showed weight loss and intermittent vomiting and dyspnea for 1 month. Therefore, the owner elected euthanasia.

Localized HSs that develop from a single region are locally invasive and tend to metastasize to draining lymph nodes. Most localized HSs are reported in the subcutaneous tissues of the extremities [2]. The tumor masses often invade the deep dermis and underlying skeletal muscles and fascia. Furthermore, localized HS can develop from a periarticular region. Previous studies have suggested that the association with a joint is because the tumor can develop from the dendritic cells of the synovial lining [3, 7].

In a review of joint tumors in dogs, PAHS was three times more common than was synovial cell sarcoma, especially in the area of the stifle joint [4]. Furthermore, an association between PAHS in the stifle joint and previous cranial cruciate ligament disease or previous joint disease has been reported [4]. Only few reports have documented PAHS in the elbow joint; this could be because of the characteristics of the elbow joints that show relatively few ligamentous diseases [4]. In this case, the tumor site was the elbow joint, which was an uncommon site, and the only clinical sign was that of forelimb lameness and pain, without previous elbow joint disease.

HS is usually observed in middle-aged Bernese Mountain Dogs, Rottweilers, Golden Retrievers, and Flat-Coated Retrievers. Although the patient's signs can lead to a suspicion of HS, diagnostic evaluation is necessary for confirming the diagnosis. Determining the exact sublineages of dendritic cells from which HSs arise is not yet possible. However, immunohistochemical evaluation using CD18 and vimentin revealed the origin of the tumor cells, and was very helpful in differentiating these tumors from osteosarcoma, synovial cell sarcoma, and malignant fibrous histiocytomas [6, 8].

With the recent development of limb-sparing techniques using allografts and xenografts, many successful cases of treatment of distal limb neoplastic disease have been reported $[9,12]$. In the present case, although the dog showed good general health and an intact shoulder joint, the region with the tumor surrounded the elbow joint and the neoplasm affected the humerus. Therefore, amputation was a reasonable treatment choice.

A more favorable prognosis may be expected for dogs with localized HS than with disseminated HS, which is an aggressive multisystem disease with a grave prognosis. The dog in this case survived only 5 months after the amputation because although the original tumor site in the elbow was completely excised, the tumor had already metastasized to the draining lymph nodes and lungs. Many multimodal therapies, including surgical resection, chemotherapy, and radiation therapy, have been reported and may be helpful in improving the prognosis [10].

Localized HS with destructive lesions involving multiple bones of a joint and a periarticular soft-tissue mass is uncommon in dogs and is of unknown etiology. PAHS should be considered an additional differential diagnosis in middle-aged to older predisposed dog breeds, including Rottweiler, presenting with lameness when radiography reveals an aggressive joint lesion. Additional diagnostic evaluations described in this case report can be performed to make a definitive diagnosis.

\section{Acknowledgments}

This study was supported by the Basic Science Research Program through the National Research Foundation of Korea (NRF) funded by the Ministry of Science, ICT \& Future Planning (NRF-2015R1C1A1A01051759) and 2016 Research Grant from Kangwon National University (No. 520160533).

\section{References}

1. Abadie J, Hédan B, Cadieu E, De Brito C, Devauchelle P, Bourgain C, Parker HG, Vaysse A, Margaritte-Jeannin P, Galibert F, Ostrander EA, André C. Epidemiology, pathology, and genetics of histiocytic sarcoma in the Bernese mountain dog breed. J Hered 2009, 100 (Suppl 1), S19-27.

2. Affolter VK, Moore PF. Localized and disseminated histiocytic sarcoma of dendritic cell origin in dogs. Vet Pathol 2002, 39, 74-83.

3. Argyle DJ, Brearley MJ, Turek MM. Decision Making in Small Animal Oncology. 1st ed. pp. 161-170, Ames, WileyBlackwell, 2008.

4. Craig LE, Julian ME, Ferracone JD. The diagnosis and prognosis of synovial tumors in dogs: 35 cases. Vet Pathol 2002, 39, 66-73.

5. Fidel J, Schiller I, Hauser B, Jausi Y, Rohrer-Bley C, Roos M, Kaser-Hotz B. Histiocytic sarcomas in flat-coated retrievers: a summary of 37 cases (November 1998-March 2005). Vet Comp Oncol 2006, 4, 63-74.

6. Fulmer AK, Mauldin GE. Canine histiocytic neoplasia: an overview. Can Vet J 2007, 48, 1041-1043, 1046-1050.

7. Harasen GL, Simko E. Histiocytic sarcoma of the stifle in a dog with cranial cruciate ligament failure and TPLO treatment. Vet Comp Orthop Traumatol 2008, 21, 375-377.

8. Hasegawa T, Hasegawa F, Hirose T, Sano T, Matsuno $\mathbf{Y}$. Expression of smooth muscle markers in so called malignant 
fibrous histiocytomas. J Clin Pathol 2003, 56, 666-671.

9. Liptak JM, Dernell WS, Ehrhart N, Lafferty MH, Monteith GJ, Withrow SJ. Cortical allograft and endoprosthesis for limb-sparing surgery in dogs with distal radial osteosarcoma: a prospective clinical comparison of two different limb-sparing techniques. Vet Surg 2006, 35, 518533.

10. Rassnick KM, Moore AS, Russell DS, Northrup NC, Kristal O, Bailey DB, Flory AB, Kiselow MA, Intile JL. Phase II, open-label trial of single-agent CCNU in dogs with previously untreated histiocytic sarcoma. J Vet Intern Med 2010, 24, 1528-1531.

11. Shortman K, Liu YJ. Mouse and human dendritic cell subtypes. Nat Rev Immunol 2002, 2, 151-161.
12. Straw RC, Withrow SJ. Limb-sparing surgery versus amputation for dogs with bone tumors. Vet Clin North Am Small Anim Pract 1996, 26, 135-143.

13. Voegeli E, Welle M, Hauser B, Dolf G, Flückiger $\mathbf{M}$. [Histiocytic sarcoma in the Swiss population of Bernese mountain dogs: a retrospective study of its genetic predisposition]. Schweiz Arch Tierheilkd 2006, 148, 281-288. German.

14. Wellman ML, Davenport DJ, Morton D, Jacobs RM. Malignant histiocytosis in four dogs. J Am Vet Med Assoc 1985, 187, 919-921.

15. Withrow SJ, Page R, Vail DM. Withrow and MacEwen's Small Animal Clinical Oncology. 4th ed. pp. 818-821, Philadelphia, WB Saunders, 2007. 